\title{
FIBRONECTIN- AND COLLAGEN-MIMETIC LIGANDS REGULATE BONE MARROW STROMAL CELL CHONDROGENESIS IN THREE-DIMENSIONAL HYDROGELS
}

\author{
J.T. Connelly ${ }^{1, \S}$, T.A. Petrie ${ }^{2}$, A.J. García ${ }^{1,2}$ and M.E. Levenston ${ }^{3, *}$
}

\begin{abstract}
${ }^{1}$ George W. Woodruff School of Mechanical Engineering, Georgia Institute of Technology, Atlanta, GA, USA
${ }^{2}$ Wallace H. Coulter Department of Biomedical Engineering, Georgia Institute of Technology, Atlanta, GA, USA

${ }^{3}$ Department of Mechanical Engineering, Stanford University, Stanford, CA, USA

${ }^{\S}$ Current address: Centre for Cutaneous Research, Barts and the London School of Medicine and Dentistry, Queen

Mary, University of London, London, UK
\end{abstract}

\begin{abstract}
Modification of tissue engineering scaffolds with bioactive molecules is a potential strategy for modulating cell behavior and guiding tissue regeneration. While adhesion to RGD peptides has been shown to inhibit in vitro chondrogenesis, the effects of extracellular matrix (ECM)-mimetic ligands with complex secondary and tertiary structures are unknown. This study aimed to determine whether collagen- and fibronectin-mimetic ligands would retain biologic functionality in three-dimensional (3D) hydrogels, whether different ECM-mimetic ligands differentially influence in vitro chondrogenesis, and if effects of ligands on differentiation depend on soluble biochemical stimuli. A linear RGD peptide, a recombinant fibronectin fragment containing the seven to ten Type III repeats (FnIII7-10) and a triple helical, collagen mimetic peptide with the GFOGER motif were covalently coupled to agarose gels using the sulfo-SANPAH crosslinker, and bone marrow stromal cells (BMSCs) were cultured within the 3D hydrogels. The ligands retained biologic functionality within the agarose gels and promoted density-dependent BMSC spreading. Interactions with all adhesive ligands inhibited stimulation by chondrogenic factors of collagen Type II and aggrecan mRNA levels and deposition of sulfated glycosaminoglycans. In medium containing fetal bovine serum, interactions with the GFOGER peptide enhanced mRNA expression of the osteogenic gene osteocalcin whereas FnIII7-10 inhibited osteocalcin expression. In conclusion, modification of agarose hydrogels with ECMmimetic ligands can influence the differentiation of BMSCs in a manner that depends strongly on the presence and nature of soluble biochemical stimuli.
\end{abstract}

Keywords: Chondrogenesis, mesenchymal progenitors, extracellular matrix, fibronectin, collagen, tissue engineering, microenvironment.

*Address for correspondence:

Marc E. Levenston

233 Durand Building

Stanford University

Stanford, CA 94305-4038, USA

E-mail: levenston@stanford.edu
Introduction

Mesenchymal stem cells (MSCs) are an attractive cell source for tissue engineering and cell-based therapies given their multilineage differentiation potential and ability to be expanded in culture (Caplan, 1991; Pittenger et al., 1999). The repair of damaged articular cartilage is one particular application that would be well suited for MSCs as a result of the limited capacity for cartilage regeneration and the rapid dedifferentiation of chondrocytes during monolayer expansion (Benya and Shaffer, 1982). Many populations of mesenchymal progenitors such as bone marrow stromal cells (BMSCs) display chondrogenic-like differentiation in three-dimensional (3D) culture environments and in the presence of transforming growth factor- $\beta$ (TGF- $\beta$ ) family growth factors (Johnstone et al., 1998; Caterson et al., 2001; Bosnakovski et al., 2004). However, recent studies have shown that even over extended culture periods, engineered cartilage constructs derived from BMSCs have a distinct composition and inferior mechanical properties compared with those derived from native articular chondrocytes (Mauck et al., 2006; Connelly et al., 2008b). It is therefore likely that additional signals are required for complete chondrogenic differentiation and the development of a functional tissue replacement.

Interactions between cells and the extracellular matrix (ECM) provide important cues for the differentiation and development of many tissues, including cartilage. For example, integrin-mediated adhesion to fibronectin is required for precartilage condensation of limb bud cells (Bang et al., 2000), and the presence of specific fibronectin isoforms influences the extent of condensation (White et al., 2003). Interactions with Type II collagen can also enhance the in vitro chondrogenesis of BMSCs (Bosnakovski et al., 2006). Although much work is still required to fully understand the complex roles of cellmatrix interactions in chondrogenesis, it is clear that the ECM provides key signals for guiding this process.

A potential strategy for controlling cell-matrix interactions in vitro is to engineer synthetic matrices to present specific ligands in a precise manner. The incorporation of peptides containing the integrin adhesive sequence Arginine-Glycine-Aspartic acid (RGD) into non-adhesive hydrogels was reported to enhance MSC survival and osteogenic differentiation (Shin et al., 2005). However, studies in our laboratory suggest that alginate and agarose hydrogels functionalized with RGD peptides inhibit BMSC chondrogenesis (Connelly et al., 
2007; Connelly et al., 2008a). In addition to modulating integrin-mediated adhesion, engineered matrices also provide a means for introducing small molecules (Benoit et al., 2008) or larger moieties such as chondroitin sulfate (Varghese et al., 2008) that can enhance in vitro chondrogenesis. Although interactions with the RGD motif inhibit BMSC chondrogenesis, interactions with adhesive sequences that mimic other ECM proteins and target a distinct set of integrin receptors may induce different responses. For example, $\alpha 5 \beta 1$ binding to fibronectin requires a synergy site containing the "PHSRN" sequence in addition to RGD (Aota et al., 1994), and $\alpha 2 \beta 1$ binds to the "GFOGER" motif within fibrillar collagens (Reyes and Garcia, 2003). However, the effects of ECM-mimetic ligands with complex secondary and tertiary structures on chondrogenesis are unknown. The goals of this study were to determine whether fibronectin- and collagen-mimetic ligands, targeting the $\alpha 5 \beta 1$ integrins and $\alpha 2 \beta 1$ integrins, respectively, retain biologic functionality when conjugated to $3 \mathrm{D}$ agarose hydrogels, whether interactions with different ECM-mimetic ligands differentially influence BMSC chondrogenesis in 3D hydrogels, and whether the responses to such engineered matrices depend on the nature of soluble biochemical stimuli presented through the culture medium.

\section{Materials and Methods}

\section{Materials}

The synthetic peptides GRGDSP(RGD) and the non-adhesive control GRGESP (RGE) were obtained from Bachem (King of Prussia, PA, USA), and the collagen mimetic peptide GGYGGGPC[GPP]5GFOGER[GPP]5GPC (GFOGER) was prepared by the Emory University Microchemical Facility. The sulfo-SANPAH crosslinker, EZ-link Maleimide-PEG-Biotin kit, and Ultralink Immobilized Avidin were from Pierce (Rockford, IL, USA). Seaprep agarose was from Cambrex (Frederick, MD, USA). Immature bovine hind limbs were from Research 87 (Marlborough, MA, USA). Recombinant human TGF- $\beta 1$ was from R\&D Systems (Minneapolis, MN, USA), and basic-fibroblast growth factor (bFGF) was from Peprotech (Rocky Hill, NJ, USA). Dexamethasone, 1,9 dimethyl methylene blue (DMMB), agarase, and Hoechst dye 33258 were from Sigma Aldrich (St Louis, MO, USA). The ITS+ premix and Proteinase K were from BD Biosciences (San Jose, CA, USA). Fetal bovine serum was from Hyclone (Logan, UT, USA). Dulbecco's modified Eagle medium (DMEM), antibiotic/antimycotic, trypsin, nonessential amino acids (NEAA), and phosphate buffered saline (PBS) were from Invitrogen (Carlsbad, CA, USA). The radiolabel precursor ${ }^{35} \mathrm{~S}$-sodium sulfate was from MP Biomedicals (Irvine, CA, USA). The ${ }^{3} \mathrm{H}$-proline and ECF substrate were purchased from GE Healthcare (Piscataway, NJ, USA). The anti-vinculin and anti-biotin antibodies were from Sigma-Aldrich, and the FITC anti-mouse antibody was from Abcam (Cambridge, UK). AlexaFluor 594-conjugated phalloidin was from Molecular Probes (Eugene, OR, USA). The RNeasy mini kit was from Qiagen (Valencia, CA,
USA), and the AMV reverse transcriptase kit was from Promega (Madison, WI, USA). The Sybr Green master mix was from Applied Biosystems (Foster City, CA, USA), and the primers were from Invitrogen.

\section{Preparation of ligand-modified agarose}

A monobiotinylated fibronectin fragment containing the seventh to tenth Type III repeats (FnIII7-10) of human fibronectin was expressed in JM109 cells and purified by affinity chromatography as previously described (Petrie et al., 2006). A biotin tag was coupled to the cysteines in the GFOGER peptide using the EZ-link Maleimide-PEGBiotin kit according to the manufacturer's instructions. The synthetic peptides and fibronectin fragment were then conjugated to agarose hydrogels with the heterobifunctional sulfo-SANPAH crosslinker (Dodla and Bellamkonda, 2006). Briefly, the primary amines on the ligands were reacted with the NHS-ester group of the sulfo-SANPAH in PBS at room temperature for $4 \mathrm{~h}$ in the dark with a 10 -fold molar excess of the crosslinker. Seaprep agarose solutions were prepared in $\mathrm{Ca}^{2+} / \mathrm{Mg}^{2+}$-free PBS, autoclaved, and cooled to $37^{\circ} \mathrm{C}$. One part peptide/sulfo-SANPAH solution was combined with three parts $4 \%$ agarose and mixed thoroughly to yield a $3 \%$ agarose solution. The mixture was exposed to $365 \mathrm{~nm}$ ultraviolet light for $3 \mathrm{~min}$ to activate the photoreactive groups of the sulfo-SANPAH and conjugate the peptide to $\mathrm{CH}$ groups in the agarose. The agarose was allowed to gel at $4{ }^{\circ} \mathrm{C}$ for $20 \mathrm{~min}$ and washed four times with fivefold excess PBS over 3 days to remove the unbound peptide and crosslinker. Controls were prepared as stated previously but without addition of the sulfo-SANPAH. Final ligand densities in the agarose gels were determined by dot blot detection of biotinylated ligands (Fig. 1). Gels were digested with agarase (4 U/ gel) at $45^{\circ} \mathrm{C}$ for $4 \mathrm{~h}$ and blotted onto nitrocellulose ( $n=3 /$ condition). Membranes were probed with alkaline phosphatase-conjugated anti-biotin antibodies and developed with the ECF substrate and Fuji Image Analyzer (Fuji, Tokyo, Japan). For an input concentration of $8 \mu \mathrm{M}$ (400 $\mu \mathrm{g} / \mathrm{mL})$ FnIII7-10, the conjugation efficiency was approximately $30 \%$, resulting in a final density of 2.4 $\mu \mathrm{M}$ (Fig 1C). For an input density of $37 \mu \mathrm{M}(200 \mu \mathrm{g} / \mathrm{mL})$ GFOGER, the conjugation efficiency was approximately $20 \%$, resulting in a final density of $7.4 \mu \mathrm{M}$ (Fig. 1C). Low levels (less than $5 \%$ ) of the ligands were present in gels without the sulfo-SANPAH crosslinker. For reference, the conjugation efficiencies for the fibronectin- and collagenmimetic ligands were slightly higher than those previously reported for laminin-1 (Dodla and Bellamkonda, 2006).

\section{Cell seeding and gel culture}

Bone marrow was harvested from the tibiae and femora of an immature calf as previously described (Connelly et al., 2007). The adherent BMSCs were expanded three times in low-glucose DMEM supplemented with $10 \%$ fetal bovine serum (FBS) and $1 \mathrm{ng} / \mathrm{mL}$ bFGF. The $3 \%$ modified agarose gels were melted at $45{ }^{\circ} \mathrm{C}$ and cooled to $37^{\circ} \mathrm{C}$. BMSCs were then seeded into cylindrical ( $4 \mathrm{~mm}$ diameter) gels by resuspending the cells in the melted agarose at a density of $10 \mathrm{e} 6 \mathrm{cells} / \mathrm{mL}$ and casting the cell suspension into 
a: FnIII7-10 Density

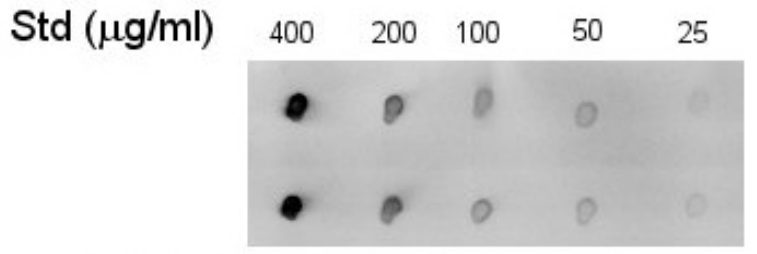

FnIII7-10 + sSANPAH
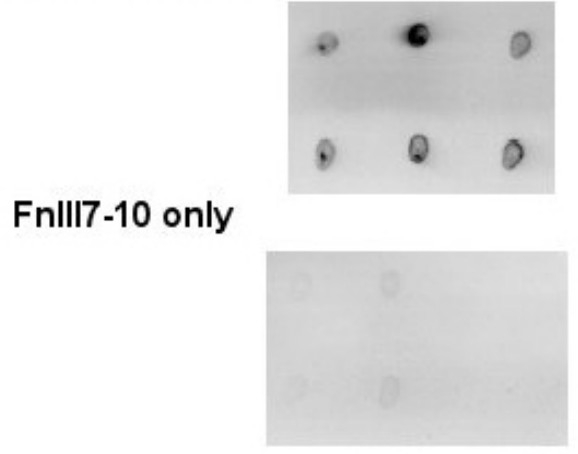

\section{b: GFOGER Density}

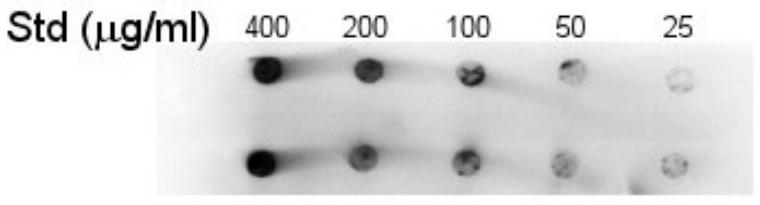

GFOGER+sSANPAH

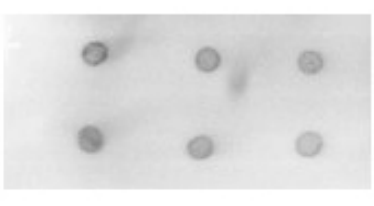

GFOGER only

\section{c: Conjugation Efficiency}

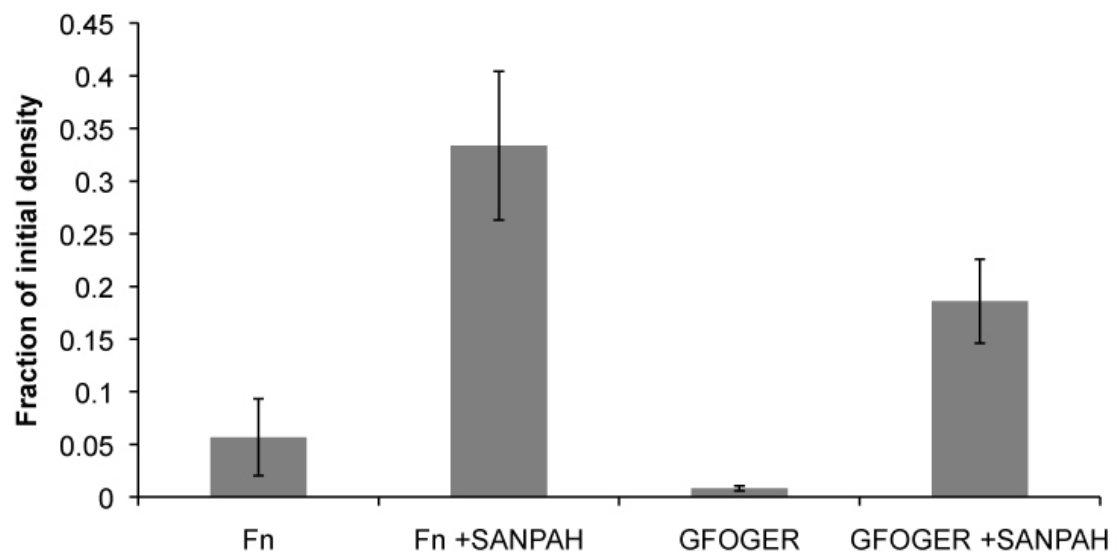

Fig. 1. Conjugation of FnIII7-10 and GFOGER ligands to agarose hydrogels. (a) FnIII7-10 and (b) GFOGER densities in the agarose gels were determined by dot blot detection of the biotin tag after agarase digestion. The initial concentration of FnIII7-10 and GFOGER were $400 \mu \mathrm{g} / \mathrm{mL}(8 \mathrm{mM})$ and $200 \mu \mathrm{g} / \mathrm{mL}(37 \mathrm{mM})$, respectively, and the standards were diluted from the ligand stocks. Control gels were prepared with the same initial ligand density but without the sulfo-SANPAH crosslinker. (c) Conjugation efficiency for the FnIII7-10 and GFOGER modified gels was quantified by measuring the dot blot intensities relative to the initial densities. For the concentrations examined, there was a linear relationship between biotin intensity and ligand concentration. Data represent the mean $\pm \mathrm{SEM}, n=3$ gels per condition.

custom molds. The gels were cooled at $4{ }^{\circ} \mathrm{C}$ for $30 \mathrm{~min}$ and transferred to fresh culture medium. The basal, chemically defined medium consisted of high-glucose DMEM, $1 \%$ ITS + Premix, $1 \%$ NEAA, $1 \%$ antibiotic/antimycotic, and $50 \mu \mathrm{g} / \mathrm{mL}$ ascorbate. The chondrogenic medium was basal medium supplemented with $10 \mathrm{ng} / \mathrm{mL}$ TGF- $\beta 1$ and $100 \mathrm{nM}$ dexamethasone. The FBS-supplemented medium consisted of DMEM, $10 \%$ FBS, $1 \%$ NEAA, $1 \%$ antibiotic/ antimycotic, and $50 \mu \mathrm{g} / \mathrm{mL}$ ascorbate. BMSC seeded gels were cultured up to 8 days under the specified conditions and media were changed every 2 days.

\section{Ligand functionality}

To examine the functionality of the ligands in the 3D hydrogels, BMSCs were seeded into agarose gels functionalized with the RGD peptide $(0,50$, and $100 \mu \mathrm{M})$, FnIII7-10 fragment $(0,0.5$, and $1 \mu \mathrm{M})$, GFOGER peptide $(0,3.75,15 \mu \mathrm{M})$, or nonadhesive RGE peptide $(100 \mu \mathrm{M})$. Cells in gels were cultured for $24 \mathrm{~h}$ in basal medium and examined for changes in cell morphology. Agarose gels were fixed with $10 \%$ neutral-buffered formalin for 30 min at $4{ }^{\circ} \mathrm{C}$ and rinsed in PBS. Portions of the fixed gels were blocked with $5 \%$ FBS and permeabilized with $1 \%$ Triton-X100. Vinculin localization was examined by staining with the anti-vinculin antibody $(10 \mu \mathrm{g} / \mathrm{mL})$ for $90 \mathrm{~min}$ at room temperature and secondary detection was performed with the anti-mouse FITC-labeled antibody $(10 \mu \mathrm{g} / \mathrm{mL})$ for $90 \mathrm{~min}$ at room temperature. The F-actin cytoskeleton was visualized by staining with AlexaFluor594-phalloidin for $90 \mathrm{~min}$ at room temperature, 
a: Cell Morphology
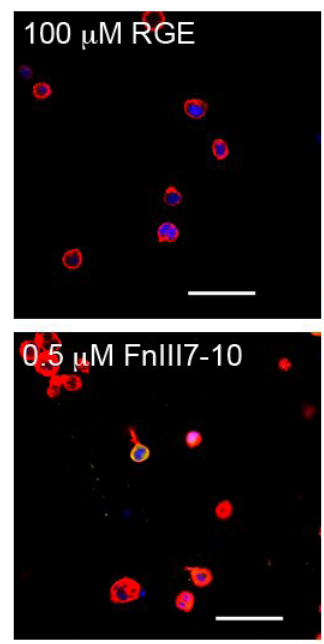
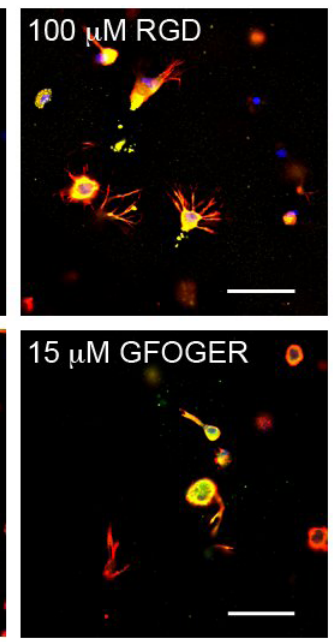

b: Cell Spreading

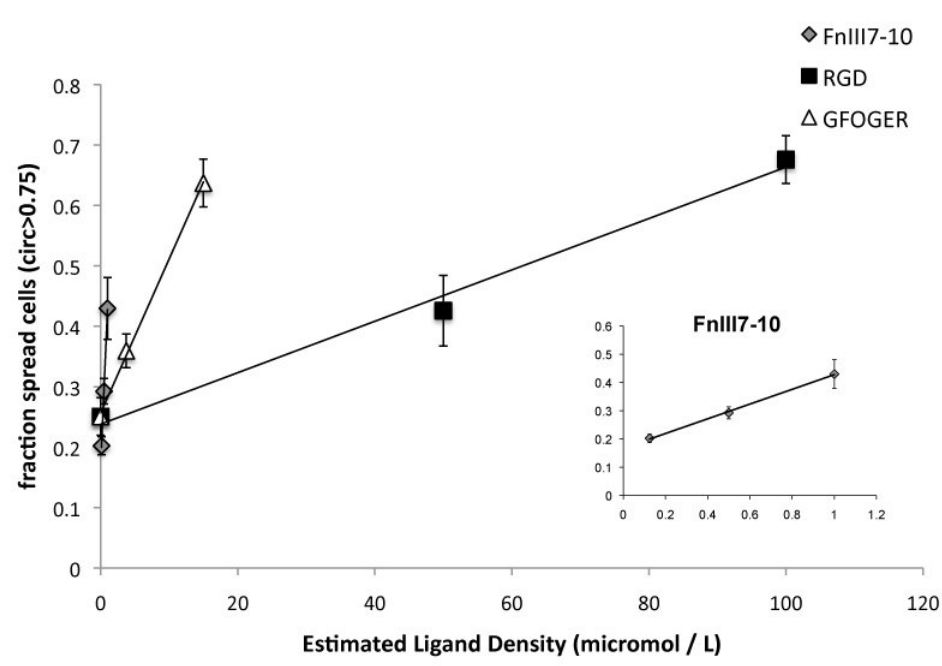

Fig. 2. Effects of ligand-modified agarose on bone marrow stromal cell (BMSC) morphology. (a) BMSC morphology was examined $24 \mathrm{~h}$ after seeding into functionalized gels by immunofluorescence detection of the F-actin cytoskeleton (red), vinculin (green), and DNA (blue). Agarose gels were modified with $100 \mu \mathrm{M}$ RGE, $100 \mu \mathrm{M}$ RGD, $0.5 \mu \mathrm{M}$ FnIII7-10, and $15 \mu \mathrm{M}$ GFOGER (final densities). Scale bars are $50 \mu \mathrm{m}$. (b) Three-dimensional cell morphology was quantified by measuring the fraction of spread cells (circularity greater than 0.75 ) over a range of ligand densities for RGD, FnIII7-10 (inset), and GFOGER. Data represent the mean \pm SEM, $n=6$ (Original magnification, 10x).

and DNA was labeled with Hoechst dye. The agarose gels were imaged with a Zeiss 510 laser scanning confocal microscope (Zeiss, Heidelberg, Germany). Fluorescence images of the F-actin cytoskeleton from two different gels (three images per gel) were analyzed with Scion Image software (Frederick, MD, USA). The area (A) and perimeter $(\mathrm{P})$ for each individual cell were used to calculate the circularity (circularity $=4 \pi A / P^{2}$ ) as a quantitative measure of cell spreading with a lower circularity indicating a greater degree of spreading (Connelly et al., 2008a).

\section{Effects of ligands on BMSC differentiation}

To investigate the effects of these ligand-functionalized gels on chondrogenic differentiation, BMSCs were first seeded into RGE $(100 \mu \mathrm{M})$, RGD $(100 \mu \mathrm{M})$, FnIII7-10 (1 $\mu \mathrm{M})$, or GFOGER $(15 \mu \mathrm{M})$ modified agarose, cultured for 8 days in basal or chondrogenic medium, and examined for changes in matrix synthesis rates and accumulation $(n=5 /$ group). To determine the lineage-specific effects of the gels, BMSCs were again seeded into RGE, RGD, FnIII7-10, or GFOGER modified agarose with the same ligand densities as in the chondrogenic experiment, cultured for 8 days in chondrogenic or serum supplemented medium, and examined for changes in chondrocytic and osteoblastic gene expression ( $n=4$ /group).

During the final $24 \mathrm{~h}$ of culture, $5 \mu \mathrm{Ci} / \mathrm{mL}^{35} \mathrm{~S}$-sodium sulfate and $10 \mu \mathrm{Ci} / \mathrm{mL}^{3} \mathrm{H}$-proline were included in the culture medium to measure sulfated glycosaminoglycan (sGAG) and protein synthesis, respectively. At the end of the culture period, radiolabel incorporation was quenched with four sequential $30 \mathrm{~min}$ washes in PBS plus $0.8 \mathrm{mM}$ sodium sulfate and $1.6 \mathrm{mM}$ L-proline at $4{ }^{\circ} \mathrm{C}$. The agarose gel constructs were weighed wet, lyophilized, and sequentially digested with Proteinase $\mathrm{K}$ (1 mg/80 mg sample) at $60{ }^{\circ} \mathrm{C}$ overnight and agarase (4 $\mathrm{U} /$ construct) at $45^{\circ} \mathrm{C}$ for $4 \mathrm{~h}$. Radiolabel contents were measured using a liquid scintillation counter. The sGAG contents were measured using the 1,9-dimethylmethylene blue assay (Farndale et al., 1982), and the DNA contents were measured using the Hoechst dye assay (Kim et al., 1988). Total collagen content was measured using the hydroxyproline assay (Woessner, 1961).

RNA was isolated from the agarose gels using the Tri-spin method (Chomczynski and Sacchi, 1987). Gels were immediately dissociated in lysis buffer containing 2-mercaptoethanol. RNA was extracted from the gel using the Trizol reagent and chloroform and precipitated with $100 \%$ isoproponol. The RNA was further purified using the Qiagen RNeasy kit according to the manufacturer's protocol. Total RNA $(1 \mu \mathrm{g})$ was reverse-transcribed to cDNA using the Promega AMV reverse transcriptase kit. Gene expression was measured by real-time reverse transcription-polymerase chain reaction (PCR) using the SybrGreen master mix and custom primers for bovine collagen II, aggrecan, collagen I (Brodkin et al., 2004), and osteocalcin (Bosnakovski et al., 2005). The PCR reactions and detection were performed with an ABI Prism 7700 (Applied Biosystems) and the transcript number relative to total RNA was calculated based on standards of known concentrations run on the same plate.

\section{Data analysis}

All data are presented as the mean \pm SEM (standard error of the mean) of representative experiments. Gene expression levels were transformed by optimal Box-Cox transformation to improve normality (Sokal and Rohlf, 1995). The effects of ligands in the presence or absence 

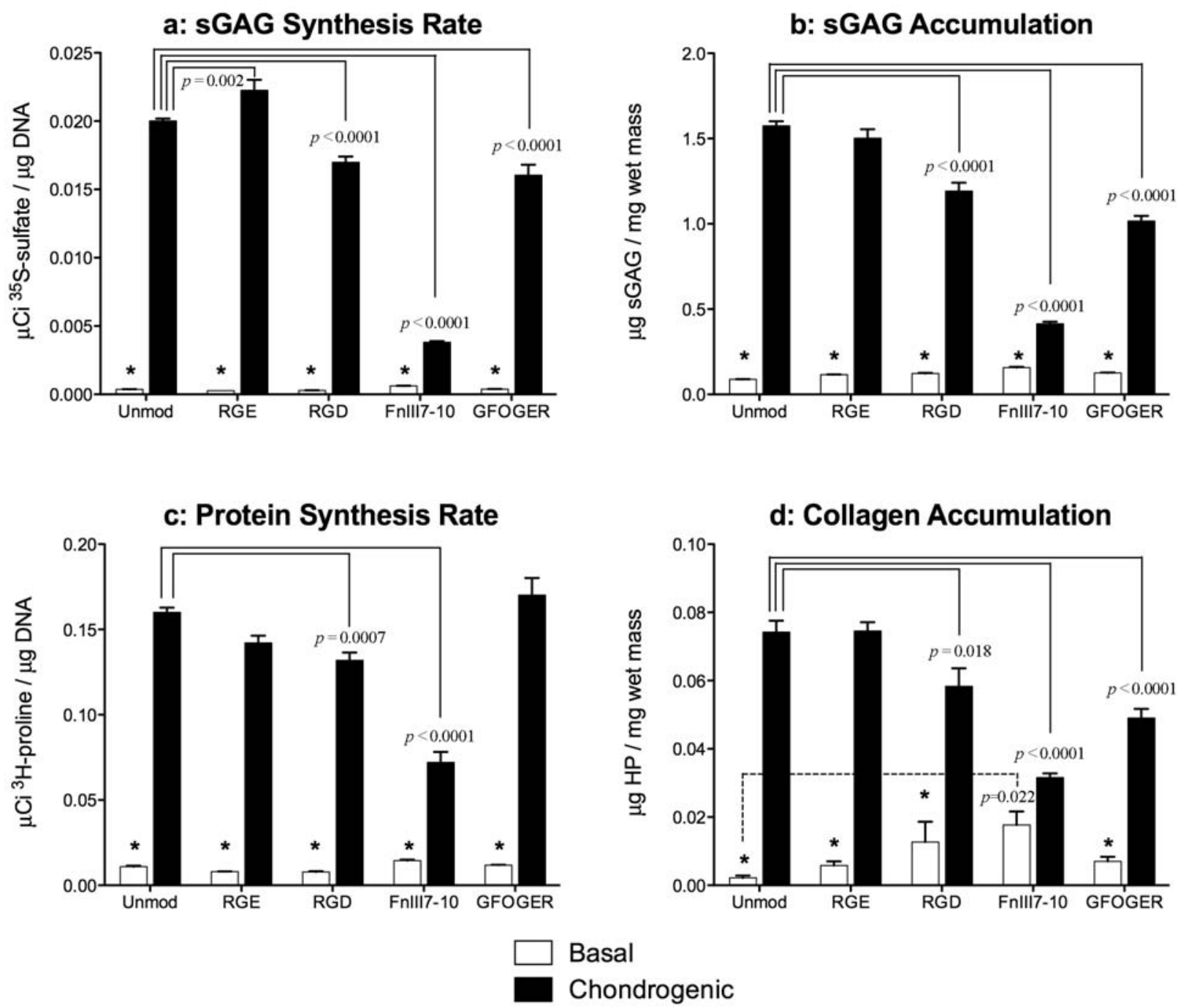

Fig. 3. Regulation of matrix accumulation by ligand-modified agarose over 8 days of culture in basal or chondrogenic medium (10 ng/mL TGF- $\beta 1$ and $100 \mathrm{nM}$ dexamethasone). (a) Proteoglycan synthesis as measured by incorporation of ${ }^{35} \mathrm{~S}$-sulfate over the final $24 \mathrm{~h}$ of culture (Day 7-8) for unmodified and functionalized agarose with $100 \mu \mathrm{M}$ RGE, $100 \mu \mathrm{M}$ RGD, $1 \mu \mathrm{M}$ FnIII7-10, or $15 \mu \mathrm{M}$ GFOGER final ligand densities. (b) Total sGAG accumulation in the gels after the 8-day culture period as measured by the DMMB assay. (c) Protein synthesis as measured by incorporation of ${ }^{3} \mathrm{H}$-proline over the final $24 \mathrm{~h}$ of culture; and (d) total collagen accumulation as measured by assaying for hydroxyproline (HP) content. Data represent the mean \pm SEM, $n=4-6$ gels/condition. Significance $v s$. chondrogenic for a given gel is indicated by $*(p<0.0001)$ or $+(p<0.001)$.

of chondrogenic supplements on matrix synthesis rates and sGAG accumulation were examined using two factor (ligand, medium) general linear models with Tukey's test for pairwise comparisons. The effects of ligands on chondrocytic and osteoblastic gene expression in chondrogenic or serum-supplemented medium were examined using two factor (ligand, medium) general linear models with Tukey's test for pairwise comparisons. Significance was at $p<0.05$.

\section{Results}

Integrin adhesive ligands alter BMSC morphology in 3D agarose gels

Like the linear RGD peptide, the recombinant fibronectin fragment FnIII7-10 and collagen-mimetic peptide containing the GFOGER sequence retained biologic functionality after conjugation to the agarose. After $24 \mathrm{~h}, \mathrm{BMSCs}$ in the non-adhesive RGE hydrogels maintained a rounded morphology. In contrast, cells in hydrogels conjugated with RGD, FnIII7-10, and GFOGER demonstrated marked changes in morphology characterized by large $\mathrm{F}$-actin projections and positive staining for vinculin (Fig. 2A), consistent with integrinmediated adhesion to the hydrogel. For each of the adhesive molecules, there was a monotonic increase in the number of spread cells with increasing ligand density (Fig. 2B). On a molar basis, FNIII7-10 was the most effective at inducing cell spreading, whereas RGD was the least effective. Similar changes in cell morphology at $24 \mathrm{~h}$ were observed for $100 \mu \mathrm{M}$ RGD, $1 \mu \mathrm{M}$ FnIII7-10, and $15 \mu \mathrm{M}$ GFOGER. 

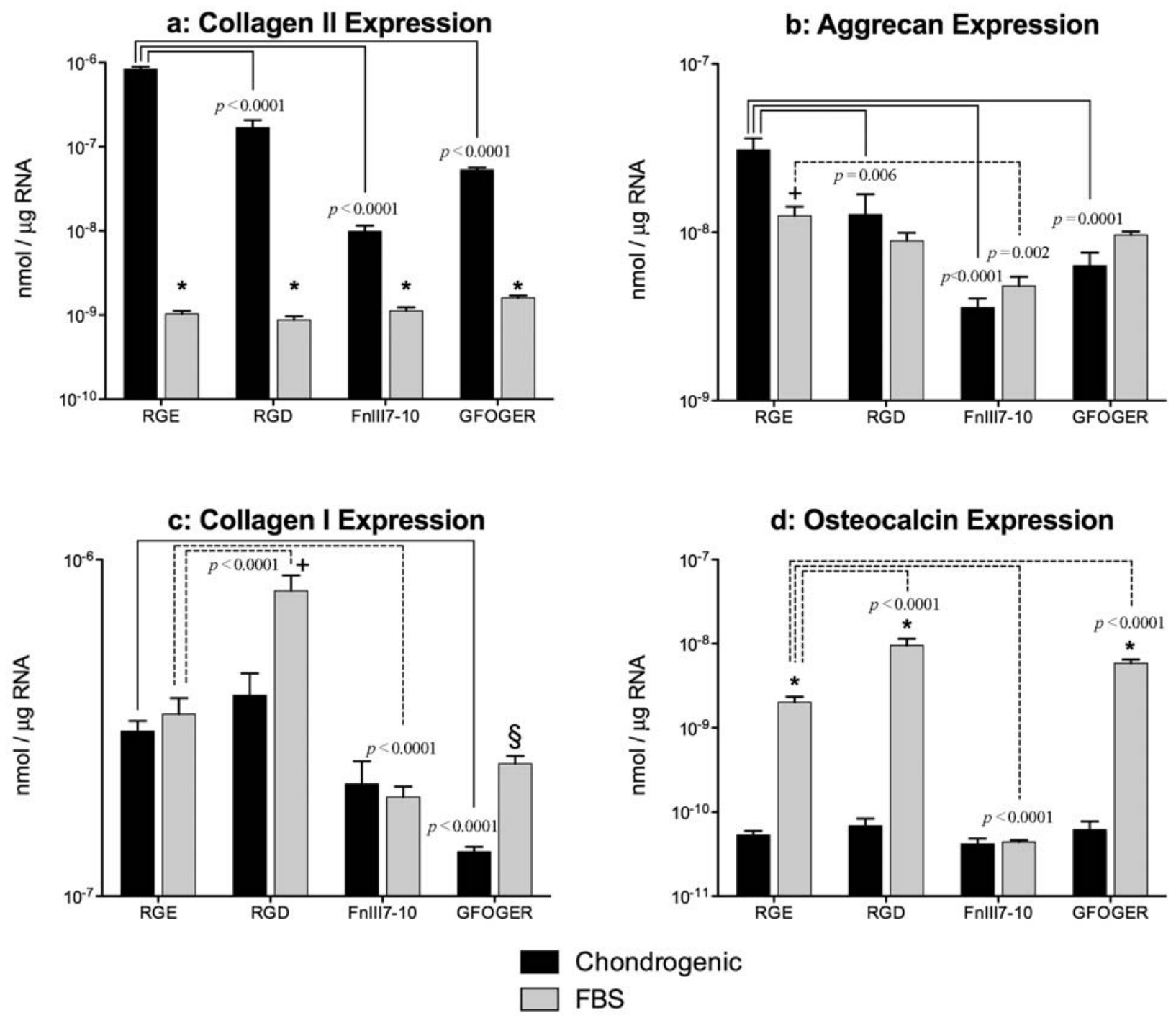

Fig. 4. Effects of ligand modified agarose on lineage-specific gene expression. Levels of mRNA for the chondrocytic genes (a) Type II collagen and (b) aggrecan were measured by real-time polymerase chain reaction after 8 days of culture in RGE, RGD, FnIII7-10, or GFOGER modified agarose. Gels were cultured in chondrogenic medium or basal medium supplemented with $10 \%$ FBS. Expression of (c) Type I collagen and the osteogenic marker (d) osteocalcin were also measured at day 8. Data represent the mean \pm SEM, $n=4-6$ gels/condition, and are presented on a logarithmic scale. Significance $v s$. chondrogenic for a given gel is indicated by $*(p<0.0001),+(p<0.001)$ or $\S(\mathrm{p}<0.05)$.

\section{Interactions with FnIII7-10 and GFOGER inhibit} matrix production during BMSC chondrogenesis

At concentrations inducing comparable degrees of cell spreading at $24 \mathrm{~h}$, all three adhesive ligands inhibited stimulation of ECM production by chondrogenic medium. Compared to basal medium, chondrogenic medium stimulated sGAG synthesis on day 8 (as measured by ${ }^{35} \mathrm{~S}$-sulfate incorporation) and sGAG accumulation within the agarose gels over the 8 day culture (both $p<0.0001$ ). Compared with unmodified gels, these responses were both inhibited by the presence of RGD, FnIII7-10 or GFOGER (all $p<0.0001$ ) (Fig. 3A,B). The chondrogenic medium also stimulated overall protein synthesis on day 8 (as measured by ${ }^{3} \mathrm{H}$-proline incorporation) and collagen accumulation over the 8 day culture (both $p<0.0001$ ). Compared with unmodified gels, interactions with RGD $(p=0.0007)$ or FnIII7-10 $(p<0.0001)$ inhibited protein synthesis, and interaction with RGD ( $p=0.018)$, FnIII7-10 $(p<0.0001)$, or GFOGER $(p<0.0001)$ inhibited collagen accumulation (Fig. 3C,D). The RGE peptide did not substantially alter matrix synthesis rates or accumulation, as only the sGAG synthesis rate was significantly different from unmodified gels $(p=0.002)$. The presence of FnIII7-10 substantially increased $(p=0.022)$ collagen deposition in basal medium (Fig. 3D).

\section{Interactions with functionalized hydrogels influence lineage-specific gene expression in a context dependent manner}

The effects of the adhesive ligands on BMSC gene expression differed depending on the biochemical cues present in the culture medium. After 8 days of culture in the presence of the chondrogenic stimuli TGF- $\beta 1$ and dexamethasone, interactions with RGD, FnIII7-10 
or GFOGER all inhibited mRNA levels (Fig. 4A-B) of the chondrocytic genes Type II collagen (all $p<0.0001$ ) and aggrecan $(p=0.0064, p<0.0001$ and $p=0.0001$, respectively). In contrast, expression of collagen II was substantially lower after 8 days of culture in $10 \%$ FBSsupplemented medium than in chondrogenic medium for all gel groups (all $p<0.0001$ ) and was not significantly affected by the presence of any of the adhesive ligands. In serum-supplemented medium, expression of aggrecan was significantly lower than in chondrogenic medium for RGE-modified gels only ( $p=0.009)$, and was inhibited by interactions with FnIII7-10 $(p=0.0018)$. Type I collagen expression (Fig. 4C) did not significantly differ between chondrogenic and serum-supplemented medium in gels modified with RGE or FnIII7-10, but was significantly greater in serum-supplemented medium in gels modified with $\operatorname{RGD}(p=0.002)$ or GFOGER $(p=0.025)$. Relative to RGE-modified gels, collagen I expression was stimulated only by interactions with RGD in serum-supplemented medium $(p<0.0001)$, and was inhibited by interactions with FnIII7-10 in serum-supplemented medium and GFOGER in chondrogenic medium (both $p<0.0001$ ). Expression of the osteogenic marker osteocalcin (Fig. 4D) was substantially higher in serum-supplemented medium compared with the chondrogenic medium in gels modified with RGE, RGD or GFOGER (all $p<0.0001$ ), and did not significantly vary among ligands in chondrogenic medium. In serum-supplemented medium, osteocalcin expression was enhanced by RGD ( $p<0.0001)$ or GFOGER ( $p=$ 0.0011 ) interactions and inhibited by FnIII7-10 interactions $(p<0.0001)$.

\section{Discussion}

Our data demonstrate that ECM-mimetic ligands with complex secondary and tertiary structures can be coupled to agarose hydrogels and retain their biologic activity. Similar to short, RGD containing oligopeptides (Connelly et al., 2008a), GFOGER and FnIII7-10 promoted densitydependent changes in the three-dimensional morphology of BMSCs. On stimulation with the chondrogenic factors TGF- $\beta 1$ and dexamethasone, the presence of each of the adhesive ligands inhibited matrix synthesis and chondrocytic gene expression relative to non-adhesive controls. Several studies found that adhesion to short RGD peptides inhibits the chondrogenesis of BMSCs (Connelly et al., 2007; Salinas and Anseth, 2008) and that this effect is mediated by the actin cytoskeleton (Connelly et al., 2008a). Our data provide additional support for the role of the cytoskeleton and suggest that cell shape, more so than the specific integrin ligand, is a key regulator of chondrogenesis. Similarly, surface chemistry has less of an effect on human MSC chondrogenesis than overall morphology and cell aggregation (Phillips et al., 2010).

In contrast, the RGD and GFOGER ligands increased expression of osteocalcin, an osteoblastic gene, in the presence of $10 \%$ FBS, whereas the FnIII7-10 inhibited this response. Taken together, these findings indicate that the specific responses of BMSCs to cues supplied by engineered three-dimensional matrices depend on the biochemical environment. Although cell shape and cytoskeletal tension also control osteogenic versus adipogenic differentiation (McBeath et al., 2004; Engler et al., 2006), our findings and others further suggest that osteogenic differentiation may depend on the specific ECM and integrins involved (Keselowsky et al., 2005). It is interesting to note that the RGD peptide, but not GFOGER or FnIII7-10, increased collagen I expression in the serum supplemented medium. These results raise the intriguing possibility that different components of the ECM may regulate different aspects of osteoblastic differentiation.

When coupled to biomaterial surfaces, the FnIII7-10 and GFOGER ligands enhance the osteoblastic differentiation of rat MSCs in vitro and the osseointegration of titanium screws in vivo (Reyes et al., 2007; Petrie et al., 2008). Although the effects of the GFOGER modified gels on osteocalcin expression in the present study are consistent with these findings, the inhibitory effects of FnIII7-10 appear somewhat contradictory. Such differences may reflect variations in the specific media formulation, which have the potential to alter cellular responses to biomaterials. For example, the presence of a phosphate source, which was absent in our culture medium, may impact the extent of osteogenesis. Alternatively, altered presentation of the FnIII7-10 between two-dimensional and three-dimensional may influence the activity and integrin specificity of the ligand. Much work is clearly still required to understand the complex and context-dependent roles of cell-ECM interactions in differentiation, and new insights in this area could lead to improved biomaterials and regenerative therapies.

Although functionalizing hydrogels with ECM mimetic ligands may be detrimental for in vitro chondrogenesis, this strategy may be advantageous for other tissue engineering applications. Adhesion to RGD peptides can enhance initial cell viability within gels, and the incorporation of enzymatically cleavable tethers allows these ligands to be removed during later stages of differentiation (Salinas and Anseth, 2008; Kloxin et al., 2009). Patterning of adhesive ligands may also allow for undifferentiated progenitors to be retained within specified regions of an engineered tissue. Finally, graded levels of bioactive molecules could be a useful strategy for engineered heterogeneous tissues or interfaces such as fibrocartilage or osteochondral replacements, where controlling the extent of the chondrogenic response to initial biochemical cues may allow the development of tissues with desired spatial patterns of phenotypic heterogeneity.

\section{Acknowledgments}

This work was supported primarily by funding (MEL) from the Georgia Tech/Emory Center (GTEC) for the Engineering of Living Tissues, an ERC Program of the National Science Foundation under Award Number EEC9731643 and was also supported by the National Institutes of Health through R01EB004496 (AJG). 


\section{References}

Aota S, Nomizu M, Yamada KM (1994) The short amino acid sequence Pro-His-Ser-Arg-Asn in human fibronectin enhances cell-adhesive function. J Biol Chem 269: 24756-24761.

Bang OS, Kim EJ, Chung JG, Lee SR, Park TK, Kang SS (2000) Association of focal adhesion kinase with fibronectin and paxillin is required for precartilage condensation of chick mesenchymal cells. Biochem Biophys Res Commun 278: 522-529.

Benoit DS, Schwartz MP, Durney AR, Anseth KS (2008) Small functional groups for controlled differentiation of hydrogel-encapsulated human mesenchymal stem cells. Nat Mater 7: 816-823.

Benya PD, Shaffer JD (1982) Dedifferentiated chondrocytes reexpress the differentiated collagen phenotype when cultured in agarose gels. Cell 30: 215-224.

Bosnakovski D, Mizuno M, Kim G, Ishiguro T, Okumura M, Iwanaga T, Kadosawa T, Fujinaga T (2004) Chondrogenic differentiation of bovine bone marrow mesenchymal stem cells in pellet cultural system. Exp Hematol 32: 502-509.

Bosnakovski D, Mizuno M, Kim G, Takagi S, Okumura M, Fujinaga T (2005) Isolation and multilineage differentiation of bovine bone marrow mesenchymal stem cells. Cell Tissue Res 319: 243-253.

Bosnakovski D, Mizuno M, Kim G, Takagi S, Okumura M, Fujinaga T (2006) Chondrogenic differentiation of bovine bone marrow mesenchymal stem cells (MSCs) in different hydrogels: influence of collagen type II extracellular matrix on MSC chondrogenesis. Biotechnol Bioeng 93: 1152-1163.

Brodkin KR, Garcia AJ, Levenston ME (2004) Chondrocyte phenotypes on different extracellular matrix monolayers. Biomaterials 25: 5929-5938.

Caplan AI (1991) Mesenchymal stem cells. J Orthop Res 9: 641-650.

Caterson EJ, Nesti LJ, Li WJ, Danielson KG, Albert TJ, Vaccaro AR, Tuan RS (2001) Three-dimensional cartilage formation by bone marrow-derived cells seeded in polylactide/alginate amalgam. J Biomed Mater Res 57: 394-403.

Chomczynski P, Sacchi N (1987) Single-step method of RNA isolation by acid guanidinium thiocyanate-phenolchloroform extraction. Anal Biochem 162: 156-159.

Connelly JT, Garcia AJ, Levenston ME (2007) Inhibition of in vitro chondrogenesis in RGD-modified three-dimensional alginate gels. Biomaterials 28: 1071 1083.

Connelly JT, Garcia AJ, Levenston ME (2008a) Interactions between integrin ligand density and cytoskeletal integrity regulate BMSC chondrogenesis. J Cell Physiol 217: 145-154.

Connelly JT, Wilson CG, Levenston ME (2008b) Characterization of proteoglycan production and processing by chondrocytes and BMSCs in tissue engineered constructs. Osteoarthritis Cartilage 16: 1092-1100.

Dodla MC, Bellamkonda RV (2006) Anisotropic scaffolds facilitate enhanced neurite extension in vitro. $\mathrm{J}$ Biomed Mater Res A 78: 213-221.
Engler AJ, Sen S, Sweeney HL, Discher DE (2006) Matrix elasticity directs stem cell lineage specification. Cell 126: 677-689.

Farndale RW, Sayers CA, Barrett AJ (1982) A direct spectrophotometric microassay for sulfated glycosaminoglycans in cartilage cultures. Connect Tissue Res 9: 247-248.

Johnstone B, Hering TM, Caplan AI, Goldberg VM, Yoo JU (1998) In vitro chondrogenesis of bone marrowderived mesenchymal progenitor cells. Exp Cell Res 238: 265-272.

Keselowsky BG, Collard DM, Garcia AJ (2005) Integrin binding specificity regulates biomaterial surface chemistry effects on cell differentiation. Proc Natl Acad Sci U S A 102: 5953-5957.

Kim YJ, Sah RL, Doong JY, Grodzinsky AJ (1988) Fluorometric assay of DNA in cartilage explants using Hoechst 33258. Anal Biochem 174: 168-176.

Kloxin AM, Kasko AM, Salinas CN, Anseth KS (2009) Photodegradable hydrogels for dynamic tuning of physical and chemical properties. Science 324: 59-63.

Mauck RL, Yuan X, Tuan RS (2006) Chondrogenic differentiation and functional maturation of bovine mesenchymal stem cells in long-term agarose culture. Osteoarthritis Cartilage 14: 179-189.

McBeath R, Pirone DM, Nelson CM, Bhadriraju K, Chen CS (2004) Cell shape, cytoskeletal tension, and RhoA regulate stem cell lineage commitment. Dev Cell 6: 483-495.

Petrie TA, Capadona JR, Reyes CD, Garcia AJ (2006) Integrin specificity and enhanced cellular activities associated with surfaces presenting a recombinant fibronectin fragment compared to RGD supports. Biomaterials 27: 5459-5470.

Petrie TA, Raynor JE, Reyes CD, Burns KL, Collard DM, Garcia AJ (2008) The effect of integrinspecific bioactive coatings on tissue healing and implant osseointegration. Biomaterials 29: 2849-2857.

Phillips JE, Petrie TA, Creighton FP, Garcia AJ (2010) Human mesenchymal stem cell differentiation on self-assembled monolayers presenting different surface chemistries. Acta Biomater 6: 12-20.

Pittenger MF, Mackay AM, Beck SC, Jaiswal RK, Douglas R, Mosca JD, Moorman MA, Simonetti DW, Craig S, Marshak DR (1999) Multilineage potential of adult human mesenchymal stem cells. Science 284: 143-147.

Reyes CD, Garcia AJ (2003) Engineering integrinspecific surfaces with a triple-helical collagen-mimetic peptide. J Biomed Mater Res A 65: 511-523.

Reyes CD, Petrie TA, Burns KL, Schwartz Z, Garcia AJ (2007) Biomolecular surface coating to enhance orthopaedic tissue healing and integration. Biomaterials 28: 3228-3235.

Salinas CN, Anseth KS (2008) The enhancement of chondrogenic differentiation of human mesenchymal stem cells by enzymatically regulated RGD functionalities. Biomaterials 29: 2370-2377.

Shin H, Temenoff JS, Bowden GC, Zygourakis K, Farach-Carson MC, Yaszemski MJ, Mikos AG (2005) Osteogenic differentiation of rat bone marrow stromal cells cultured on Arg-Gly-Asp modified hydrogels without 
dexamethasone and beta-glycerol phosphate. Biomaterials 26: $3645-3654$.

Sokal RR, RohlfFJ (1995) Biometry: the principles and practice of statistics in biological research. W.H. Freeman, New York.

Varghese S, Hwang NS, Canver AC, Theprungsirikul P, Lin DW, Elisseeff J (2008) Chondroitin sulfate based niches for chondrogenic differentiation of mesenchymal stem cells. Matrix Biol 27: 12-21.

White DG, Hershey HP, Moss JJ, Daniels H, Tuan RS, Bennett VD (2003) Functional analysis of fibronectin isoforms in chondrogenesis: Full-length recombinant mesenchymal fibronectin reduces spreading and promotes condensation and chondrogenesis of limb mesenchymal cells. Differentiation 71: 251-261.

Woessner JF, Jr. (1961) The determination of hydroxyproline in tissue and protein samples containing small proportions of this imino acid. Arch Biochem Biophys 93: 440-447.

\section{Discussion with Reviewers}

Reviewer I: There appears to be an increase in collagen production in basal conditions in FN-modified gels. Please comment. Is this type I or type II collagen? Was any immunostaining performed to verify that gene expression changes were reflected in matrix accumulation and type? Authors: As additional patterns among ligands in basal medium may have been obscured by the large difference in matrix production between chondrogenic and basal medium, we re-examined the matrix synthesis rates and matrix accumulation for constructs in basal conditions only by means of a one factor general linear model with Tukey's test for pairwise comparisons. Indeed, the FnIII7-10-conjugated group was the only group to demonstrate collagen accumulation significantly greater than that in unmodified gels in basal medium ( $p=0.0053)$. Furthermore, the sGAG accumulation, sGAG synthesis rate, and protein synthesis rate were all significantly greater in FnIII7-10-conjugated gels than in any other group in basal medium $(p<0.0001, p<0.0001$ and $p=0.0011$, respectively). The patterns of response to ligands in basal medium thus appear to be roughly opposite to those in chondrogenic medium (Fig. 3). It is tempting to ascribe these effects to differences among ligands, perhaps related to the specific integrins involved in adhesion to the scaffold. However, while the ligand concentrations were chosen based on comparable effects on cell spreading at $24 \mathrm{~h}$, it is also possible that similar effects on matrix production would be seen for the other ligands at higher densities. This issue certainly merits exploration in future studies.

We previously observed that interactions with RGDmodified alginate inhibited both collagen II gene expression and collagen II deposition by BMSCs (Connelly et al., 2007, text reference), and that cyclic tensile loading of BMSCs in fibrin enhanced both collagen I gene expression and collagen I deposition (Connelly et al., 2010, additional reference). We would thus expect a general correspondence between gene expression and protein deposition patterns in the current study, but did not examine at the protein level the specific collagen types produced in the different gels.

Reviewer I: What would the effects be over a longer time course as the cells accrete their own pericellular matrix? Also, does cell spreading continue or does the $24 \mathrm{~h}$ time point represent the steady state condition? This would be important in interpreting the longer-term responses, given the authors finding that shape is such a strong determinant of phenotypic conversion.

Authors: Cell spreading persists for at least one week, but beyond this point production of a pericellular matrix likely would interfere with access to the engineered ligands. We previously observed that cells in RGD-modified alginate gels accumulated a fibronectin-rich extracellular matrix within 6 days (Connelly et al., 2008a, text reference) and also observed that sGAG synthesis rates in the RGDmodified and unmodified gels were similar after two weeks in culture, although total sGAG accumulation still lagged in the RGD-modified gels (unpublished). These results suggest that the chondrogenic supplements are sufficient stimuli to eventually promote chondrogenesis in ligandfunctionalized gels, although it is not yet clear whether the cells and matrix will eventually catch up or if the initial cell-scaffold interactions are sufficient to permanently alter the cell phenotype or behavior.

Reviewer I: Were any measures of viability taken in these different contexts (i.e., with FN and GFOGER ligands)? Does having any attachment improve overall cell health/ viability as it appears to do with RGD?

Authors: We did not examine cell viability in this study, although we did examine the total DNA content of the gels. As expected, DNA content was significantly greater in chondrogenic medium than in basal medium for all ligand groups (all $p<0.0001$ ). Among basal medium groups, RGD- and RGE-conjugated gels had higher DNA contents than unmodified, GFOGER- or FnIII7-10-conjugated gels (all $p<0.0001$ ). Among chondrogenic groups, all conjugated gels had lower DNA contents than unmodified gels $(p \leq 0.018)$, and the DNA contents of GFOGER- and FnIII7-10-conjugated gels were significantly lower than those of all other groups (all $\mathrm{p}<0.0001$ ). Consistent with the effects on matrix production and gene expression, interactions with the integrin ligands generally inhibit the cellular response to the chondrogenic supplements.

Reviewer I: It is not clear how many times this work was repeated. Is this the result of a single donor? If so, have other replicates been performed to validate the findings?

Authors: The results presented involve cells from a single bovine donor, and describe one experiment examining matrix synthesis and accumulation and a second, independent experiment examining gene expression, with good agreement between the two studies. Additionally, patterns of sGAG synthesis and accumulation were similar in two other related experiments, which consistently showed inhibition by all ligands. The effects of RGDinteraction are similar to our previous findings involving RGD-modified alginate and agarose (Connelly et al., 2007; 
Connelly et al., 2008a, text references). Furthermore, we have recently observed reduced chondrogenic gene expression and matrix production by human BMSCs in (adhesive) fibrin gels relative to (non-adhesive) alginate gels (unpublished). We are thus fairly confident in our general findings. The issue of donor variability is an important one of substantial relevance to clinical implementation of stem cell-based therapies, but will be more appropriately explored for human donors.

Reviewer II: The main results the authors present demonstrate that these ligands are not suitable for $3 \mathrm{D}$ chondrogenesis in hydrogels. Their discussion of the likely reasons is sound and reasonable, but is it likely that anybody would wish to attempt this approach? There are some potentially interesting findings in relation to the osteogenesis specific genes. However, much further work would be warranted to elucidate the mechanisms of action. Please comment!

Authors: We believe that the results of this study are interesting and relevant for several reasons. First, assuming that the only relevant goal is the generation of an engineered articular cartilage, information on strategies that do not produce a favorable outcome are just as valuable as information on strategies producing a desired effect. Results indicating that a strategy does not "work," particularly if they arise from well controlled, carefully executed studies, are quite important to the field as a whole. A focus purely on what "worked," rather than on understanding how or why one approach works while another does not, was an endemic problem in the early development of tissue engineering as a discipline.

Second, we believe that these results are quite relevant. Adhesive scaffolds (both engineered and natural proteinbased) are widely used in tissue engineering studies, including many studies on chondrogenesis. Our results are highly relevant to understanding cell behaviors in a wide range of scaffolds currently under investigation and may aid in the improvement of those scaffolds or understanding the tradeoffs involved in using such scaffolds. The fact that ligands targeting different integrins all inhibit chondrogenesis suggests that this should be a general concern for chondrogenesis in any scaffold material providing integrin binding domains. Furthermore, identification of aspects of the cellular environment that inhibit chondrogenesis has bearing on understanding potential reasons for inadequate chondrogenesis in clinical treatments such as microfracture.

Third, we disagree with the notion that approaches that do not directly enhance the extent of hyaline-like chondrogenesis are inherently not useful or interesting, as there are situations in which inhibition of chondrogenesis may in fact be a desirable or necessary outcome. For example, the cells from fibrocartilaginous tissues such as the knee meniscus, temporomandibular joint disc, acetabular labrum, and annulus fibrosus are similar to, but not identical to, articular chondrocytes, and furthermore display notable regional heterogeneity. Most current tissue engineering approaches to such tissues are similar to those employed for articular cartilage, and do not involve approaches designed to specifically promote fibrocartilaginous differentiation. Understanding how to tune the chondrogenic differentiation of progenitors with instructive scaffolds may in fact be quite useful for engineering non-hyaline cartilages.

Regarding the effects on osteogenic genes, we agree that this is interesting (as noted in the discussion). However, exploration of the differential effects of different ligands on osteogenesis is a separate issue well beyond the scope of this study.

\section{Additional Reference}

Connelly JT, Vanderploeg EJ, Mouw JK, Wilson CG, Levenston ME (2010) Tensile loading modulates bone marrow stromal cell differentiation and the development of engineered fibrocartilage constructs. Tissue Eng Part A 16: 1913-1923. 